\title{
Experimental Study on Double Driven Single Direction Bending Flexible Joint
}

\author{
Yongyong Zheng ${ }^{1, \mathrm{a}}$, Dexu Geng ${ }^{2, \mathrm{~b}^{*}},{\text { Xiaomin } \text { Liu }^{2}, \text { Yunwei Zhao }}^{2}$, Hongbo Liu $^{1}$ \\ ${ }^{1}$ College of Mechanica Engineering, Beihua University, Jilin Province, People's Republic of China \\ ${ }^{2}$ Engineering Training Center, Beihua University, Jilin Province, People's Republic of China \\ azhengyongyong226@163.com , bgengdx64@163.com
}

Key word: Flexible joints, Bending deformation, Gripping force

Abstract: This paper develops a new type of pneumatic flexible joint, which is composed of flexible air bag, spring plate and restriction element, which is composed of two artificial muscles. It has a degree of freedom. In order to study the bending deformation and gripping ability of the joint, the experimental system was designed and built. The experimental results show that the joint can achieve a large bending deformation and have a large clamping force.

\section{Introduction}

Pneumatic flexible joint is a new type of pneumatic actuator with high flexibility, compact structure, simple control, low cost, safe and reliable, compared with the traditional ${ }^{[1-2]}$. These characteristics have been paid more and more attention by domestic and foreign scholars and have been developed rapidly, which has a good development prospect ${ }^{[3]}$. The first pneumatic artificial muscle was invented in 1950 by the Mckibben muscle, which pioneered the study of pneumatic artificial muscles, and has been applied to a variety of flexible joints of robot ${ }^{[4]}$. Since then, experts and scholars have developed a variety of joints, Since then, experts and scholars have developed a variety of joints, such as the production of shadow air muscles of the British company Shadow ${ }^{[5]}$ and the development of pneumatic bending joint by Libin Zhang, Qinghua Yang scholars and other research who come from Zhejiang University of Technology ${ }^{[6]}$.

All kinds of flexible joints of pneumatic flexible joints are characterized by large deformation and nonlinear ${ }^{[7]}$,Through experimental research and theoretical analysis, the domestic and foreign scholars have carried out a lot of research on the mechanical properties and theoretical model and achieved good results ${ }^{[8-9]}$. With the continuous development of the pneumatic flexible joint, the performance requirements are also increasing. In this paper, a double drive type single direction bending flexible joint is proposed, which can achieve large bending deformation, and has a large clamping force. Through the method of experimental research, this paper expounds the principle of bending deformation, and verifies that it has large bending deformation and large clamping force, which can meet the special requirements of some special occasions.

\section{Structure and principle of bending deformation of joints}

The developed pneumatic double drive type single direction bending flexible joint (Fig.1) is composed of the upper and lower end caps, the constraints, the latex tubes and the spring steel plates. The main function of the restriction is to limit the radial expansion of the latex tube through the compressed gas, The spring steel plate is arranged in the rectangular hole of the restriction element. The two ends are fixedly connected with the upper and lower ends respectively. The main function is to support the joint and the axial deformation of the joint, and the lower end cover is provided with a gas inlet. 


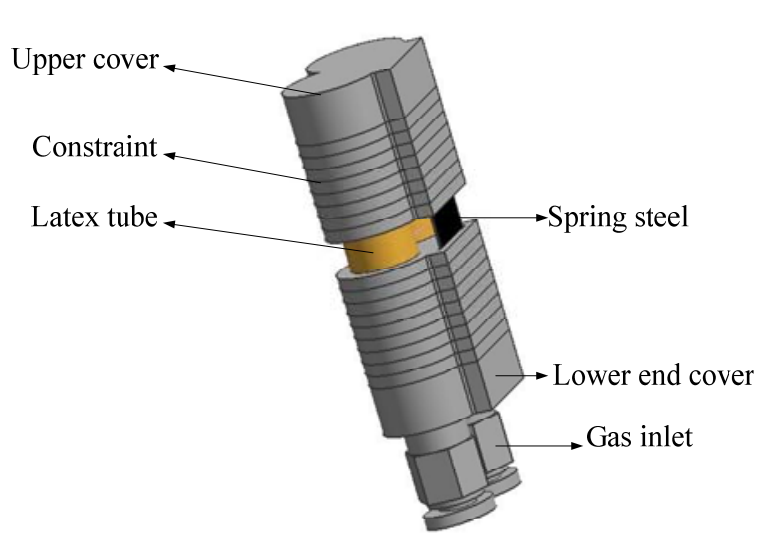

Fig. 1 Three-dimensional model of Joint

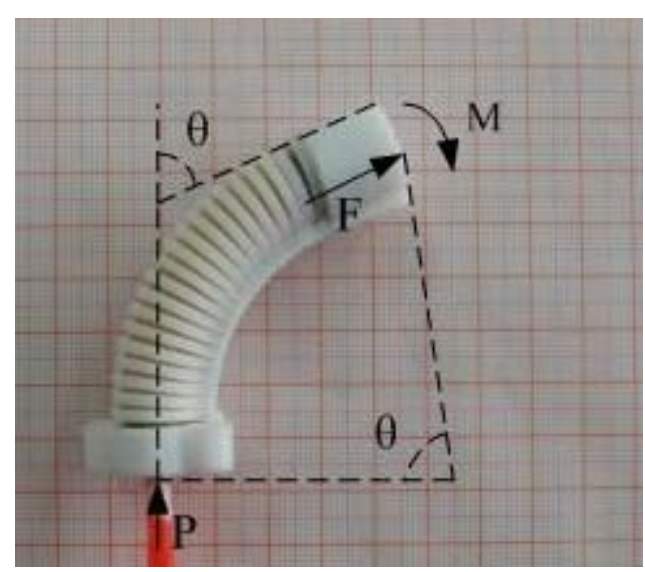

Fig. 2 Force analysis of Joint

When the pressure value is $P$, the pressure of the confined latex tube is expanded, because of the bondage of the constraints, and the axial pressure of the joint end cap is $F$, and the joint end cap is restrained by the spring steel plate, which is transformed into the bending moment $\mathrm{M}$, which makes the joint to the spring steel plate. The bending angle of the joint surface of the $\theta$ (Fig. 2) with the increase in gas pressure increases.

\section{Joint bending experiment and analysis}

\section{Experiment process}

The experimental principle of bending deformation of double driven single direction bending joint (Fig. 3), the experimental system is composed of gas source, pneumatic triple component, precision pressure reducing valve, single chip microcomputer and gyroscope (Fig. 4).

In the experimental measurements taken without contact, size by adjusting the precision pressure relief valve to control the pressure value $\mathrm{P}$, through the single chip microcomputer and the gyroscope to measure different pressure values corresponding to the joint bending angle theta. In order to reduce the influence of the measurement error on the experimental results, the average value of the experimental results is carried out under the same conditions. The main parameters of the joints are shown in Table 1.

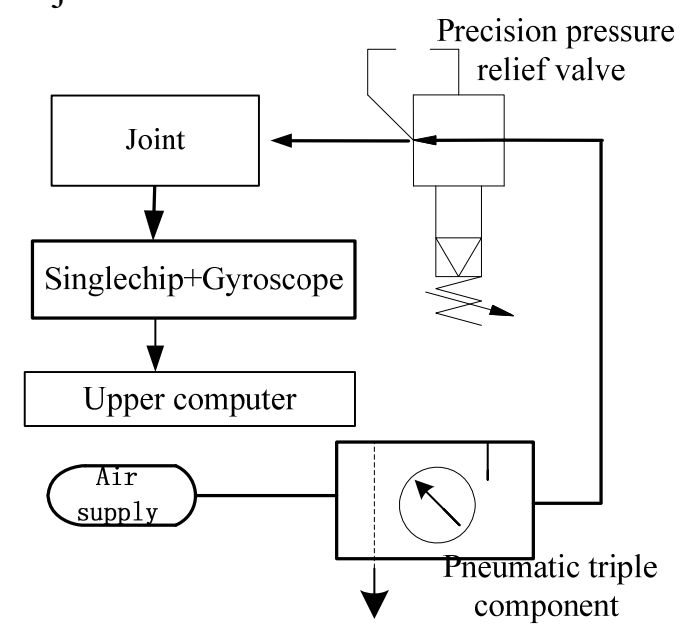

Fig. 3 The principle of experiment system

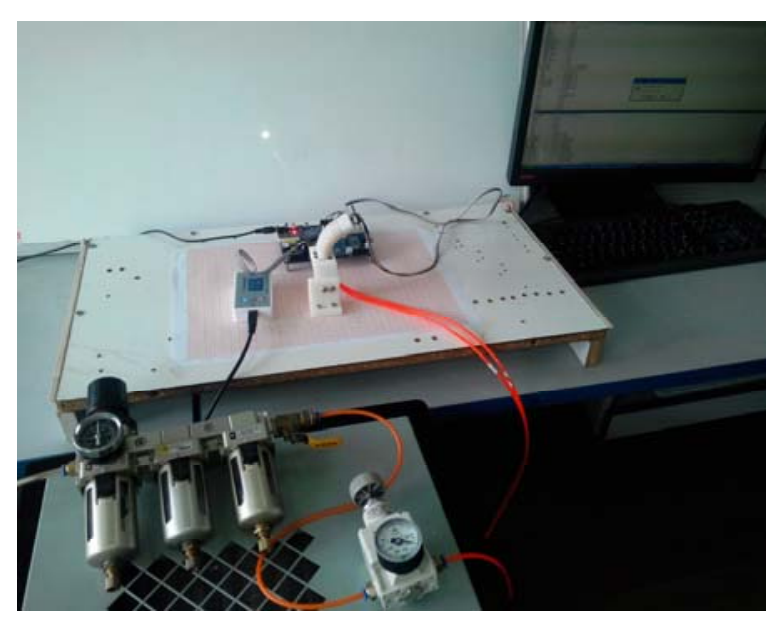

Fig.4 System of experiment 
Table 1 The Parameters of joint

\begin{tabular}{lc} 
effective length of joint & $40 \mathrm{~mm}$ \\
inner diameter of latex tube & $8 \mathrm{~mm}$ \\
outer diameter of latex tube & $11 \mathrm{~mm}$ \\
the thickness of constraints ring & $2 \mathrm{~mm}$ \\
number of constraints & 20 \\
the thickness of cover & $8 \mathrm{~mm}$ \\
thickness of spring steel & $0.25 \mathrm{~mm}$ \\
length of spring steel & $52 \mathrm{~mm}$ \\
width of spring steel & $12 \mathrm{~mm}$ \\
elastic modulus of spring steel & $206 \mathrm{GPa}$ \\
Distance from the steel plate to & \\
the center of the latex tube & $9.5 \mathrm{~mm}$ \\
\hline
\end{tabular}

\section{Experimental analysis}

The experimental data were processed with the mean value, then the curve was drawn using data processing software MATLAB, and the corresponding curve of the bending angle and air pressure was obtained (Fig. 5).

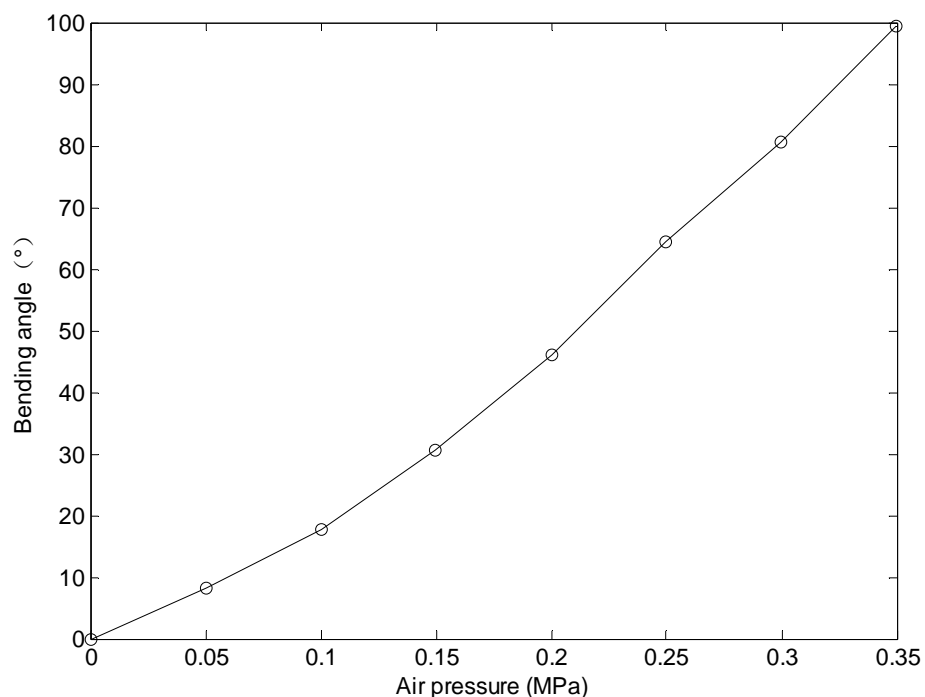

Fig. 5 The deformation curve of joint

From Fig. 5 it can be seen that the bending angle of the joint is increased with the increase of the pressure value. The bending deformation curve of the joint is nonlinear, and the main reason is that the bending deformation of latex tube is increasing. The joint can achieve a large bending deformation, when the pressure value is $0.35 \mathrm{MPa}$, the bending angle is 100 degrees.

The experimental data of the bending deformation of the double drive single direction bending joint are carried on the two polynomial fitting, and the corresponding empirical formula is obtained, which is the basis of the following research.

Bending angle equation of double driven single direction bending joint:

$$
\theta=211.1 P^{2}+217.3 P-1.227
$$

\section{Joint clamping force experiment and analysis}

\section{Experiment process}

Double drive unidirectional bending joint clamping force experiment principle (Fig. 6), the system consists of an air source, a gas dynamic triple pieces, precision pressure relief valve, XY mobile platform and digital dynamometer (Fig. 7).

Contact measurement is used in the experiment,through regulation precision pressure relief valve 
to control pressure size, by reading the digital display the number of dynamometer for joint clamping force $F_{n}$ 。 In order to reduce the influence of the measurement error on the experimental results, the average value of the experimental results is carried out under the same conditions.

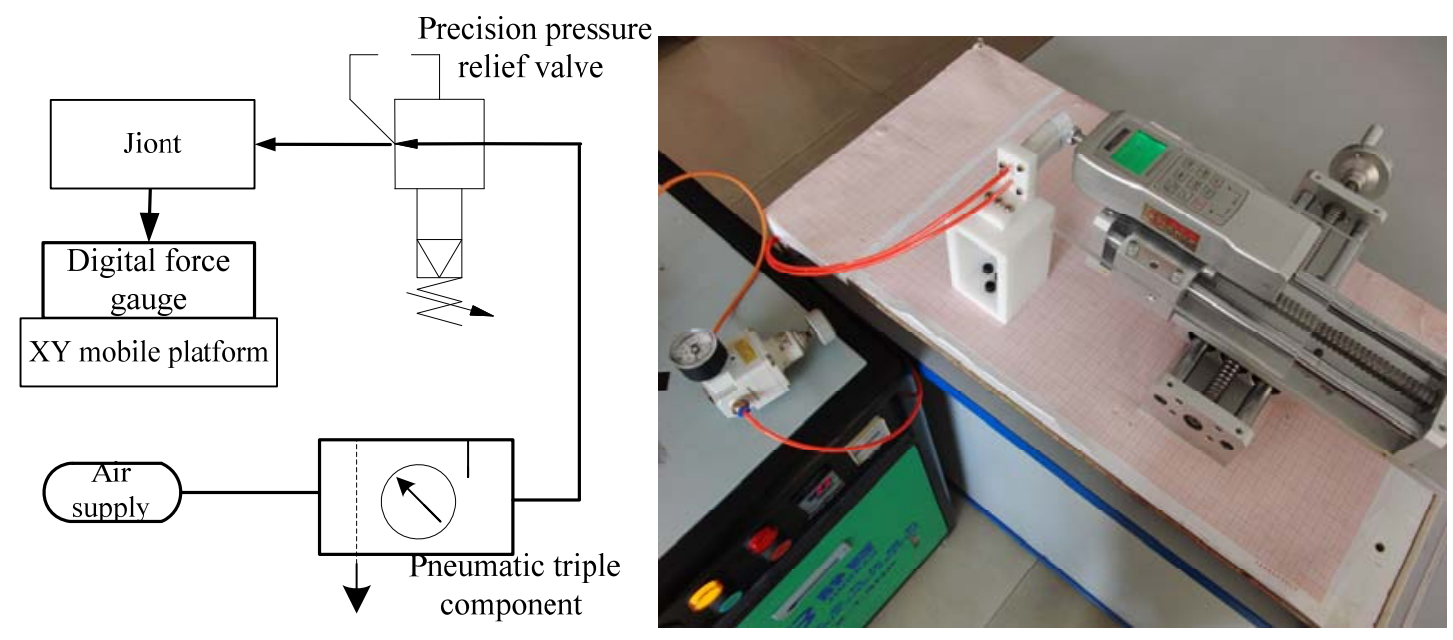

Fig. 6 The principle of experiment system

Fig. 7 System of experiment

\section{Experimental analysis}

The experimental data were processed with the mean value, then the curve was drawn using data processing software MATLAB, and the curve of the joint clamping force and the pressure value was obtained (Fig. 8).

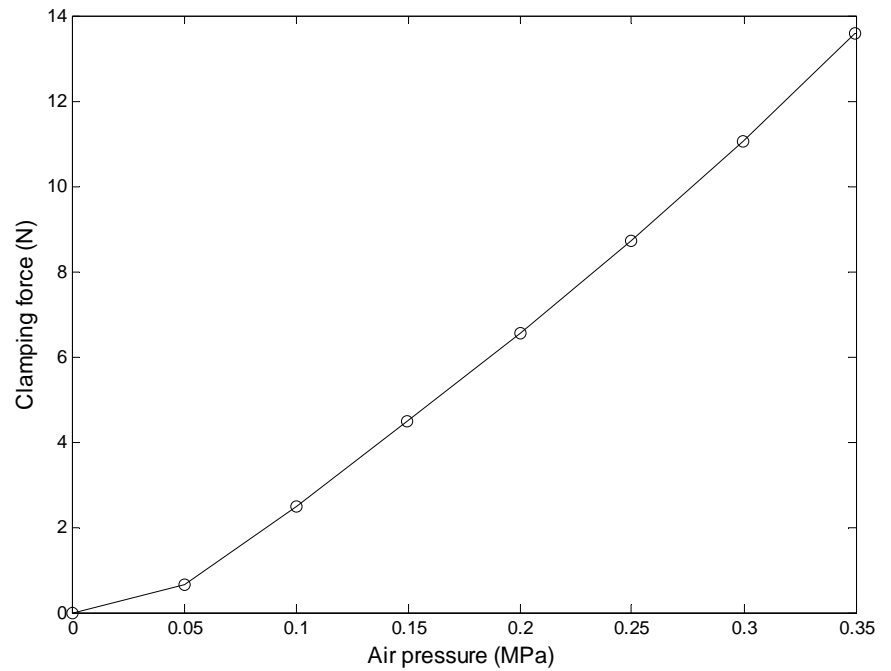

Fig. 8 The Bending deformation curve of joints under the constraints of ring surface

From figure 8, it can be seen that, with the increase of the pressure, the clamping force of the joint is gradually increasing, and the change curve of the clamping force is linear. The joint has a large clamping force and the clamping force is $13.6 \mathrm{~N}$ when the pressure value is $0.35 \mathrm{MPa}$.

The experimental data of the double drive type single direction bending of the joint force of the clamping force is carried on the two polynomial fitting, and the corresponding empirical formula is obtained, which is the basis of the following research.

Double driven single direction bending joint clamping force equation is:

$$
F_{n}=42.86 p^{2}+24.99 p-0.3112
$$




\section{Summary}

This paper developed the double drive type unidirectional bending joint of bending test and clamping force experiment. Experimental results show that the new type joint can not only achieve greater bending, but also has great blessing, at $0.35 \mathrm{Mpa}$, bending angle and clip to force respectively: $100^{\circ}$ and $13.6 \mathrm{~N}$ and meet the design requirements. In addition, the experimental data and the clamping force of the joint bending angle are two polynomial fitting, and the corresponding empirical formula is obtained, which is the basis for further study.

\section{Acknowledgements}

The project is supported by the National Natural Science Foundation (51275004), Science \& technology development project of Jilin Province(20150101026JC), Science and Technology Innovation Development Plan of Jilin City Science and Technology Bureau(201464043 \& 20156413), Youth Science Foundation Project of Jilin Provincial Science \& Technology Department(20150520109JH), and the program for the Dr. Jilin Provincial Education Department "Twelfth Five Year" science \& technology research project (20150158),PR China.

*Corresponding author: Dexu Geng, Gengdx64@163.com

\section{References}

[1]B.Gorissen, R. Donose, D. Reynaerts, et al. Procedia Engineering ,25:681-684.(2011)

[2] Dexu Geng.[D].Jilin: Jilin University (2011)

[3] Aline De Greef, Pierre Lambert, Alain Delchambre. Towards flexible medical instruments: Review of flexible fluidic actuators[J]. Precision Engineering, 33: 311-321.(2009)

[4]Shenyuan Zhang,Mingchub Liu,etc al.Hydraulic and Pneumatic.7:13-15.(2008)

[5]Shadow Robot Group (London), The SHADOW Air Muscle, $<$ http://www.shadowrobot.com/>.

[6]Pengcheng Huang,Qinghua Yang,Junguan Bao,etc al.[J].Robotics.35:67-72.(2013)

[7]Liping Yan,Peng Hou[J]. Journal of Northern Jiaotong University.26:29-33.(2002)

[8] Yoel Shapiroa,Alon Wolfa, Kosa Gaborb. Bi-bellows: Pneumatic bending actuator[J]. Sensors and Actuators A. 167:484-494.(2011)

[9]Xioamin Liu.[D].Jilin:Jilin University(2013) 